Shortage of public green spaces, functional mutations and saturation: a growing urban problem. The case of Plaza Almagro in the city of Buenos Aires

PALABRAS CLAVE - PAISAJE URBANO - ESPACIOS VERDES PÚBLICOS · PRÁCTICAS SOCIALES - USOS COTIDIANOS

KEYWORDS - URBAN LANDSCAPE · PUBLIC GREEN SPACES SOCIAL PRACTICES - EVERYDAY USES

\section{RESUMEN}

Los espacios verdes públicos urbanos contribuyen a la identidad y configuración de los paisajes barriales $y$, a la vez, son el escenario donde una numerosa y heterogénea diversidad de usuarios desarrolla sus actividades cotidianas y expresa sus intereses y tensiones respecto del área. En este sentido, el presente trabajo tiene como objetivo analizar la problemática actual de excesiva concentración de actividades y usuarios en los espacios verdes urbanos, tomando como caso de estudio La Plaza Almagro, en el barrio homónimo de la Comuna 5 de la Ciudad Autónoma de Buenos Aires, con el fin de reflexionar acerca de su rol como lugar de desarrollo de las prácticas cotidianas de los vecinos. Asimismo, se busca dar cuenta de la influencia del diseño de la Plaza en su dinámica de funcionamiento y la correlación que esto tiene con su función como elemento de cohesión social para la comunidad.

\section{ABSTRACT}

Urban public green spaces contribute to the identity and configuration of neighborhood landscapes and, at the same time, they stand as the scenario where a large and heterogeneous diversity of users develop their daily activities and express their interests and tensions regarding the area. In this sense, the present paper aims at analyzing the current problem of excessive concentration of activities and users in urban green spaces, taking as a case study Plaza the homonymous neighborhood of the Commune 5 of the Autonomous City of Buenos Aires. The purpose of this research is to reflect about its role as a place for everyday activities. Likewise it seeks to account for the influence the design of the square has in tis dynamics of $c$ design of the square has in its dyne inics of operation and the correlation that this has with its function as an element of social cohesion for the community.

\title{
Escasez de espacios verdes públicos, mutaciones funcionales y saturación: una problemática urbana creciente
}

\author{
El caso de Plaza Almagro en la ciudad de Buenos Aires
}

AGUSTINA VITTAR ·Universidad de Buenos Aires, Buenos Aires ·agusvittar_91@hotmail.com

Fecha de recepción: 01 de octubre 2019 - Fecha de aceptación: 25 de noviembre 2019

\section{INTRODUCCIÓN}

El espacio público es aquel en el que se lleva a cabo la vida colectiva de una ciudad, sobre el cual todos los habitantes tienen los mismos derechos y obligaciones. Allí es donde se va construyendo la identidad de una ciudad y de sus ciudadanos a lo largo del tiempo, y su resultado determina su imagen. Es el paisaje que da identidad y carácter a una ciudad, el que permite reconocerla y vivirla. La lógica de conformación de los espacios públicos muestra la dinámica de funcionamiento de la sociedad que los crea y habita y, por lo tanto, el espacio no existe en forma absoluta sino relativa, resultado de una producción social y como una manifestación del orden social (Páramo y Burbano, 2011).

Dentro de estos espacios, las plazas y parques se destacan por cumplir importantes funciones sociales como el encuentro entre personas, la cohesión social, la creación de comunidad, y también aquellas relacionadas con la salud física y mental de la población, la recreación y el deporte. En palabras de Jordi Borja "la organización interna del espacio urbano debe facilitar la cohesión sociocultural de las comunidades barriales, de grupos de edad, étnicas, etc". (2000, p. 161).

El área de estudio de este trabajo, Plaza Almagro, se encuentra en el barrio de Almagro, perteneciente a la Comuna 5 de la Ciudad Autónoma de Buenos Aires, y es la única área verde con tipología de "plaza" del barrio (FIGURA 1). Según los datos registrados por el Gobierno de la Ciudad de Buenos Aires (2018a) el promedio de espacios verdes públicos por habitante de la Comuna 5 es de $0.02 \mathrm{~m}^{2} / \mathrm{hab}$, lo que la posiciona como la comuna con menor cantidad de espacios verdes públicos por habitante de la ciudad, siendo el promedio de la ciudad de $0.6 \mathrm{~m}^{2} / \mathrm{hab}$. A su vez, ocupa el segundo puesto en cuanto a densidad poblacional (2018b), con $28.043 \mathrm{hab} / \mathrm{km}^{2}$, mientras que el promedio de la Ciudad Autónoma de Buenos Aires es de 14.994 $\mathrm{hab} / \mathrm{km}^{2}$.

La Plaza Almagro se ubica entre las calles Sarmiento, Bulnes, Tte. Gral. Juan D. Perón y Salguero y se encuentra rodeada también, en sus cuatro laterales, por fuertes envolventes conformados por edificios de departamentos. Tiene una superficie de 1.43 hectáreas y está organizada a partir de un área seca circular central, donde confluyen los principales senderos que comunican con los accesos a la Plaza (Figura 2). Cuenta con zona de juegos infantiles, canil, cancha multiuso y mesas de ajedrez que se ubican sobre los bordes del espacio verde, además de estos sectores con usos definidos, el resto de la Plaza es utilizada por los vecinos para múltiples actividades tanto individuales como colectivas en distintos horarios de la semana. 
1. Localización del caso de estudio Plaza Almagro. Fuente: elaboración propia en base a plano interactivo de Buenos Aires Ciudad. www.buenosaires.gob.ar/comuna-5/obras-comunales.

2. Vistas aéreas de Plaza Almagro. Fuente: Archivo de la autora. 2018.

3. Ingreso a la Plaza Almagro sobre la esquina de las calles Sarmiento y Bulnes. Actividades sobre el área central de la Plaza. Fuente: elaboración propia, 2018.

- Ciudad Autónoma de Buenos Aires (CABA)

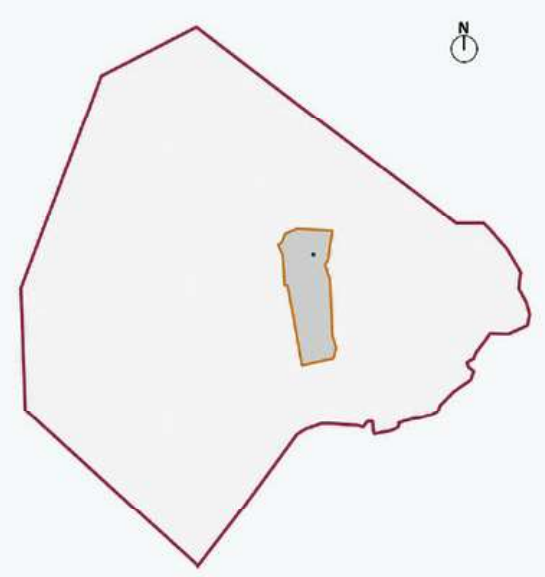

Esta Plaza es un espacio muy representativo de Almagro, inaugurada en el año 1934, ha sido testigo del desarrollo del barrio a lo largo de su historia hasta el día de hoy y se constituye como parte de una de las áreas nodales, tanto a nivel circulatorio como comercial, más relevantes de Almagro, transformándola en un lugar de referencia para los vecinos (FIGURAs 3 y 4) y punto obligado de reuniones, actividades culturales, recreativas, celebratorias y manifestaciones ciudadanas. La Plaza contribuye, así, de manera fundamental a su identidad y a la configuración de su paisaje siendo, a su vez, el soporte físico de las actividades humanas cotidianas de la comunidad barrial que la habita y la manifestación de su cultura (Gómez Carmona y Villar Calvo, 2013).

La principal problemática del caso de estudio es la superposición de actividades, debido a que varias de ellas se llevan a cabo en simultáneo en determinados horarios y en los mismos sectores de la Plaza y, al ser incompatibles, resultan en una interferencia entre sí, lo cual dificulta que los usuarios puedan desarrollarlas cómodamente. En este sentido, el objetivo del artículo es evidenciar, a través del caso de estudio de la
Comuna 5

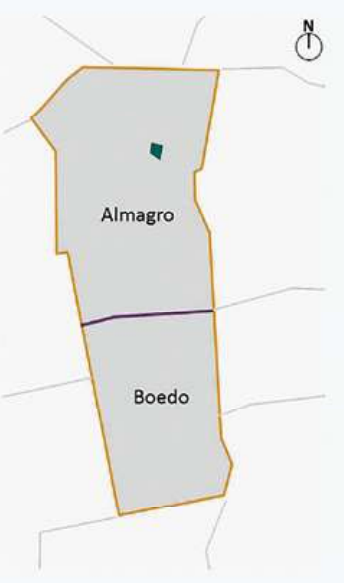

(1)

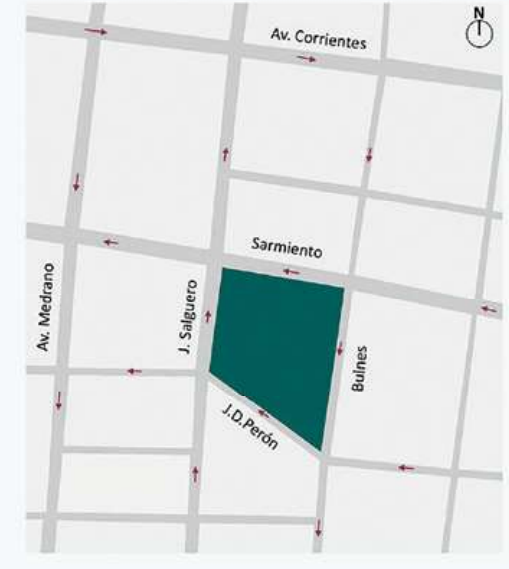

Limite CABA

Limite Comuna 5

Limite entre barrios Comuna 5

Comuna 5

Plaza Almagro
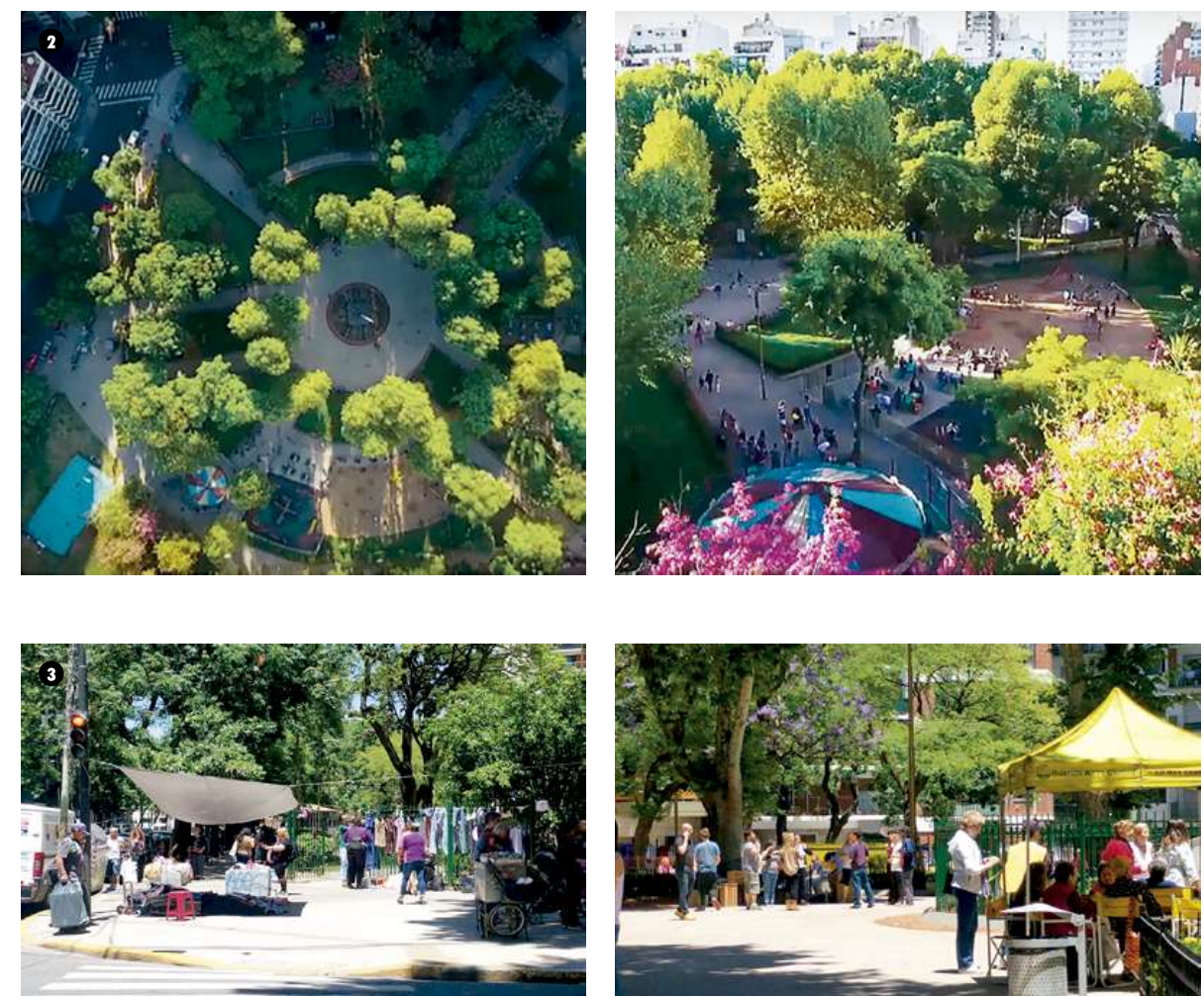
4. Sectorización Plaza Almagro. Fuente: elaboración propia. 2018.

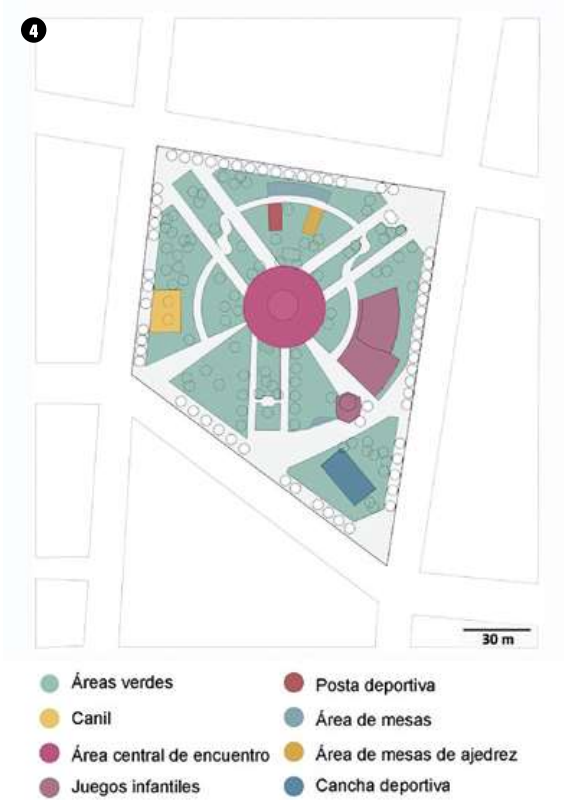

Plaza Almagro, una problemática actual que puede verse replicada en otros espacios verdes públicos de la Ciudad de Buenos Aires como lo es el sobreuso de las áreas verdes y sus cambios funcionales en relación con las dinámicas sociales presentes.

\section{METODOLOGÍA}

Según Dollfus, además de un lugar, es decir un asiento territorial, cada espacio posee una posición, que "depende del sistema de relaciones que mantiene el elemento con otros elementos, ya estén estos próximos o alejados" (1990, p.18). Por lo tanto, como primer paso en el desarrollo de la investigación, se realizó un reconocimiento del área de estudio (Plaza Almagro) y del entorno afectado por el mismo (Barrio de Almagro y Comuna 5) a través de una metodología cualitativa, el análisis de imágenes satelitales, planos, datos estadísticos y censales e información recolectada disponible en páginas web oficiales del Gobierno de la Ciudad de
Buenos Aires y Google Earth. Luego, se llevó a cabo un análisis empírico sobre las actividades realizadas en la Plaza, mediante observación participante durante distintos horarios del día y de la semana. Tanto las observaciones como la comparación de planos con el relevamiento del área, mostraron las diversas mutaciones estructurales y funcionales del paisaje (Santos, 1996). Las mutaciones estructurales hacen referencia a los cambios en sus formas ya sea por modificación, agregado o eliminación de equipamiento, cambios o desgaste de los materiales, ampliación o reducción de zonas vegetadas o zonas de material seco, entre otras; por otro lado, las mutaciones funcionales del área son los cambios en las actividades, y el ritmo de las mismas, que son llevadas a cabo en los distintos sectores de la Plaza por los usuarios. Estas observaciones llevadas a cabo para la detección de las mutaciones funcionales, se centraron en las siguientes unidades de análisis (Hernández Sampieri, Fernández-Collado, Baptista Lucio, 2006):

\section{- El ambiente social y humano: para} detectar las formas de organización en grupos y subgrupos, patrones de interacción o vinculación dentro de la Plaza, es decir, cuáles son las características de los grupos que se forman, si hay jerarquías dentro de ellos, con qué propósitos se reúnen, identificar si hay una red que los vincula, quiénes son los actores clave y cuáles son sus costumbres.

- Actividades individuales y colectivas: refiere a determinar qué acciones se realizan en la Plaza, en qué área de la misma se llevan a cabo, en qué momento del día y de la semana suceden, si se repiten de manera rutinaria o son esporádicas en el tiempo, cuál es su propósito, conocer si se realizan de manera individual o colectiva.

- Artefactos que utilizan dentro del espacio y con qué función: se busca conocer qué elementos o mobiliario utilizan los usuarios, qué funciones cubren, si la función de los artefactos dispuestos en la Plaza es la misma que efectivamente cumplen o si los usuarios hacen otro uso de ellos, si hay mobiliario que no es utilizado y, también, si falta mobiliario para la realización de algún tipo de actividad.

Por otro lado, se consultó a los usuarios de la Plaza, para lo cual se utilizó el método cuantitativo, a través de la realización de encuestas. El tamaño de la muestra fue de 50 encuestados, y se seleccionaron a usuarios de la Plaza, de diferentes edades dentro del rango 18-80+ años. El instrumento usado fue el cuestionario, que contó con seis preguntas tanto abiertas como cerradas (Duverger, 1996). A través de estas preguntas se buscó determinar si la persona encuestada es vecina del barrio de Almagro, si hace uso de la Plaza y con qué frecuencia, detectar las actividades que allí realiza para poder comprender su relación con el espacio, conocer la opinión de la persona sobre su experiencia como usuario del espacio público con relación a los distintos sectores y elementos que lo conforman y, por último, averiguar cuáles son los aspectos negativos de la Plaza que los encuestados consideran prioritarios a la hora de buscar mejoras en el funcionamiento del espacio, cuyo fin es poder revelar problemas que afectan a los usuarios y que no habían sido tenidos en cuenta.

Luego de su realización se procedió a la elaboración de sus resultados, a través de operaciones técnicas que permitieron expresarlos en porcentaje sobre la colectividad sondeada.

Con la información obtenida se confeccionó un diagnóstico cuyo producto gráfico consiste en mapeos y esquemas que facilitan su visualización y dan cuenta de la configuración actual de la Plaza y su dinámica dentro del barrio y la comuna.

\section{LA PLAZA Y SU CONTEXTO HISTÓRICO}

La Plaza de Almagro lleva el nombre del dueño de la tierra y fue producto de variadas mutaciones estructurales y funcionales (Santos, 1996) del espacio urbano. La manzana donde se encuentra hoy la Plaza estaba ocupada 
por una quinta, corralones y un tambo, cuyos terrenos fueron cedidos al Estado en 1928, aunque recién pudo ser inaugurada como "Plaza de Almagro" en 1934.

Desde la década del sesenta fue lugar residencial privilegiado por su accesibilidad al centro comercial y bancario de Buenos Aires, y su densidad poblacional y constructiva comenzó a crecer. La Plaza se constituyó así en un lugar de identidad del barrio de Almagro.

De acuerdo a la información del Gobierno de la Ciudad de Buenos Aires (2019), la Plaza Almagro fue remodelada en 2007 y en 2015. Las modificaciones de mayor importancia llevadas a cabo en el año 2007 fueron el cambio de ubicación de los juegos infantiles y el enrejamiento perimetral, que se colocó para permitir cerrarla durante horas de la noche, buscando así reducir los actos de vandalismo e inseguridad en el interior de la misma, pero que pone en riesgo su acceso libre, disfrute y uso como bien público (Narváez Muelas, 2019). Más allá de estas modificaciones, el diseño base de la Plaza se mantuvo sin cambios significativos.

Luego, hacia fines de 2015, fue intervenida para una "Puesta en valor" en la cual se renovaron los juegos infantiles.

Hoy la Plaza continúa teniendo las mismas problemáticas que antes de las reformas, pero los vecinos siguen adueñándose de este espacio público como una necesidad y un derecho ciudadano. En este sentido, cabe aclarar que la representación social de la Plaza como espacio público es una construcción colectiva e individual, es el modo en que los vecinos y usuarios la interpretan y los significados que están asociados a su imagen y a su identidad (Rey Castillo-Villar, 2015). Por lo cual, es importante tener en consideración que cada intervención sobre el espacio implica modificar algún aspecto de esa imagen que puede alterar de manera profunda la representación colectiva que tiene la comunidad sobre el mismo, como así también las representaciones individuales de cada uno de los usuarios que lo habita.

\section{FUNCIONES DE LA PLAZA}

El espacio urbano cumple funciones y ofrece servicios para sus habitantes $y$, también, para los de su entorno (Dollfus, 1990). Debido a la escasez de espacios verdes públicos en el barrio y a la alta densidad poblacional del mismo, organizada mayormente en edificios de departamentos, mencionada anteriormente, la Plaza Almagro cumple un importante rol como área de esparcimiento, donde los sujetos sociales tienden a asociarse o vincularse de forma voluntaria manifestándose relaciones de poder, alianzas y apoyo mutuo (Blanco-Bello et al., 2013).

Teniendo en cuenta el ambiente social y humano observado, se detectó que otra de sus funciones es la de punto de encuentro, ya que al ser un lugar céntrico del barrio se encuentra rodeado de distintas instituciones educativas lo que produce una gran afluencia de usuarios a la Plaza que asisten a esos lugares, en su mayoría niños y ióvenes. Por otro lado, cumple una función importante como nodo de circulación porque hay muchos medios de transporte que tienen sus paradas alrededor de la Plaza o en sus inmediaciones, como así también bicisendas, siendo un punto de gran conectividad con el resto de la ciudad. Se puede agregar la función comercial que cumplen tanto los alrededores como la Plaza en sí, con la presencia de comercios destinados al abastecimiento barrial.

Además, la Plaza como espacio público se entiende también como un espacio político, en tanto los diferentes sectores de la sociedad que convergen en él tienen posturas e intereses diferentes, que buscan instalarse y eventualmente sobreponerse a los otros (Fernández, 2013).

Con relación a la función de esparcimiento que la Plaza presenta, cuenta con áreas verdes, juegos para niños, canil para mascotas, mesas para juegos de ajedrez, estación deportiva, estación de bicicletas y una cancha deportiva. En cuanto a la función comercial, está dada por la calesita, la Feria ltinerante de Abastecimiento Barrial y los feriantes irregulares.
La intensidad de uso de los distintos sectores varía en función de las mutaciones funcionales que sufre la Plaza, es decir, de los cambios en el ritmo de los usuarios según el horario o los días de la semana, donde cada espacio se diferencia en su función dependiendo de qué manera se presentan las personas que los habitan (Santos, 1996). Las distintas intensidades de usos fueron determinadas mediante dos métodos, por un lado, la encuesta, que fue realizada a través de un cuestionario durante cinco jornadas y el tamaño de la muestra fue de 50 encuestados, todos ellos usuarios de la Plaza, de diferentes edades dentro del rango 18-80+ años. Por el otro, se hicieron observaciones durante distintos días y horarios, y en diferentes espacios de la Plaza, enfocadas en el tipo de actividades individuales y colectivas que se llevan a cabo, su forma y momento de realización, y en el uso de los artefactos presentes en el espacio y en su función, con la intención de conocer las conductas de los usuarios y el nivel de apropiación de cada sector por parte de ellos.

Durante los días de semana se observó que los usuarios permanecen durante un menor tiempo en la Plaza o la utilizan como lugar de paso, principalmente en sentido diagonal conectando las entradas ubicadas en las esquinas de las calles Sarmiento y Bulnes y Salguero y J.D. Perón. Las actividades que más se llevan a cabo son el uso de los juegos infantiles, la práctica de ejercicio físico y el paseo de mascotas (FIGURA 5). Quienes permanecen más tiempo en la Plaza lo hacen, en mayor medida, en las horas de la mañana y después de las 17 horas, debido fundamentalmente al horario laboral y escolar de los usuarios.

Los fines de semana y feriados, en cambio, la frecuencia de usuarios es mayor y, además, permanecen un tiempo más prolongado en la Plaza, siendo el caudal de usuarios similar durante todo el día, y a las actividades anteriormente mencionadas se suman otras más relacionadas con la recreación y el descanso como el uso de las áreas verdes para reuniones o "pic-nic", así como también una mayor permanencia de los niños en el área de juegos 
5. Intensidad de usos durante días de semana. Fuente: elaboración propia 2018
6. Intensidad de usos durante fines de semana feriados. Fuente: elaboración propia 2018. y de los usuarios en general en los bancos y las zonas de mesas (FIGURA 6). En estos momentos de mayor cantidad de usuarios durante un largo período de tiempo en la Plaza, se produce una superposición de usos ya que los espacios destinados a actividades específicas se ven saturados y las personas pasan a realizar esas actividades a las áreas multifunción, pero al ser escasas aquellas que se encuentran en buen estado y no ser de fácil acceso para los usuarios, el resultado es una concentración en pequeños espacios determinados, desaprovechándose así metros cuadrados de terreno potencialmente útiles.

La intensidad de usos también varía en función de la época del año, durante los meses de verano la mayor afluencia de público se da en los horarios de la mañana y de la tarde-noche, evitando el mediodía y las primeras horas de la tarde donde el asoleamiento es más fuerte. En cambio, en la época invernal el horario de mayor uso de la Plaza es más acotado y se da durante la mañana y el mediodía, antes de que el asoleamiento comience a ser más débil.

Tanto los desplazamientos, como las actividades de permanencia ancladas en un lugar, constituyen prácticas cotidianas de movilidad que se llevan a cabo, en este caso, en la Plaza Almagro que se transforma, entonces, en el escenario de rutinización de las mismas (Lindón, 2006). Siendo, por lo tanto, fundamental el análisis de las mutaciones funcionales, entendiéndolas como una diferenciación de funciones dentro del espacio resultado de los distintos modos de habitarlo según los ritmos de los usuarios, como parte de la comprensión de las dinámicas sociales propias del lugar que se ven afectadas por la incongruencia con la estructura y diseño de la Plaza.

Los usos también se fueron modificando a lo largo del tiempo debido a diversas mutaciones estructurales, es decir, cambios físicos en el aspecto del espacio (Santos, 1996), que la Plaza ha sufrido desde los años 40 hasta la actualidad, producto de distintas políticas de Estado con respecto al espacio público.

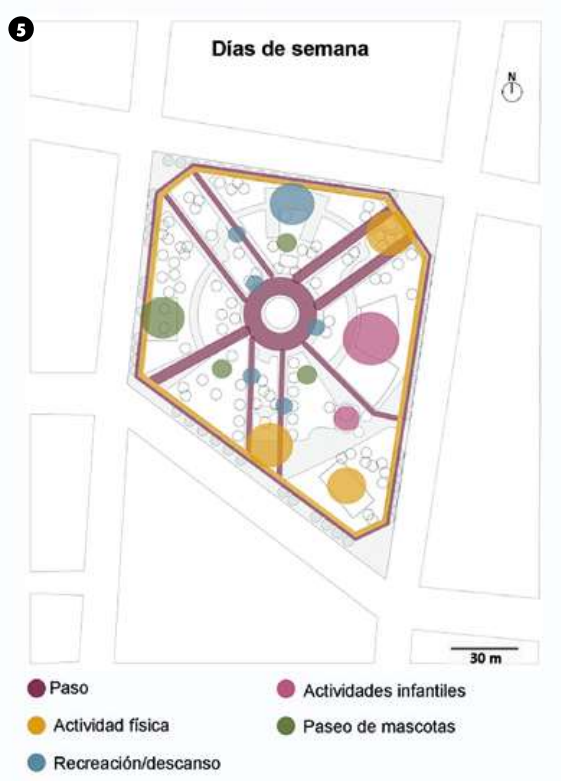

El cambio en los tipos de viviendas que la rodean, que a partir de los años 60 dejaron de ser casas bajas y comenzaron a ser edificios de departamentos, aumentó la densidad de habitantes $y$, por ende, la afluencia de usuarios a la Plaza de distintos grupos etarios y con intereses diversos también fue in crescendo; la incorporación de la reja en 2007 terminó de modificar por completo la fisonomía de la Plaza, haciendo de ella un espacio "cerrado" con horarios de apertura y cierre.

Según Santos "al ser susceptible a los cambios irregulares a lo largo del tiempo, el paisaje es un conjunto de formas heterogéneas, de edades diferentes, pedazos de tiempos históricos representativos de diversas maneras de producir las cosas, de construir el espacio" (Santos, 1996, p. 65) y esta definición representa la realidad actual de la Plaza, que es producto de todas sus mutaciones y, a la vez, es una muestra que refleja los cambios sociales, económicos y las distintas posturas políticas frente al espacio público a lo largo del tiempo desde su creación.

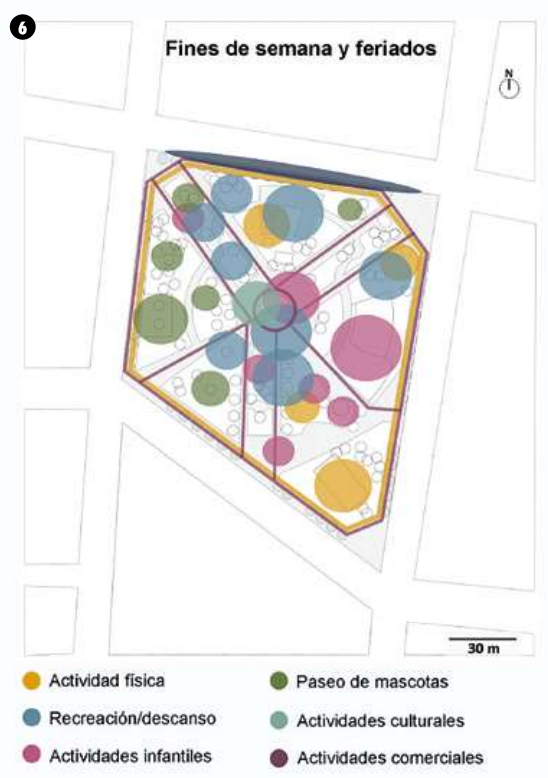

\section{ACTORES SOCIALES Y SU VÍNCULO CON LA PLAZA}

Entendiendo por actor social a quienes, con sus actividades, construyeron a lo largo de los años la identidad de la Plaza y hoy la mantienen (Jodelet, 1986) como resultado de una acción colectiva que le otorga sentido al espacio público (Anduze, 2018), se puede hablar de una importante diversidad de actores sociales intervinientes en la plaza Almagro.

El espacio adquiere un significado mediante la acción colectiva de la multidiversidad de usuarios implicados en la utilización del mismo, de manera que, a medida que lo usan, habitan, y transforman.

Los actores más influyentes en la Plaza son el Gobierno y los vecinos del barrio que realizan allí actividades. El Gobierno, en este caso de la Ciudad de Buenos Aires, es el responsable de accionar y generar transformaciones que influyen en las dinámicas de la Plaza. Por otro lado, los vecinos son quienes se apropian o no 
del espacio público, teniendo así la capacidad de reclamar cambios que se ajusten a sus necesidades dentro del mismo.

A su vez, dentro de este grupo formado por los vecinos del barrio, hay distintos tipos de actores según su rango etario y las actividades que cada uno realiza en la Plaza, por ejemplo, actividad física, ajedrez, paseo de mascotas o llevar a los niños al sector de juegos, entre otras. Esto le suma complejidad al espacio público ya que las distintas perspectivas que estos actores tienen sobre la Plaza llevan, en muchas ocasiones, a que desplieguen sus intereses contrapuestos, tanto en el uso cotidiano de la Plaza como a la hora de reclamar transformaciones para la misma.

Así, el espacio público debe ser un lugar donde esta diversidad de actores, no exenta de conflictos y desacuerdos, pueda desarrollarse sin estar obligados a ser socialmente ni culturalmente homogéneos, ni a asumir comportamientos uniformes (Roldán, 2018).

El procesamiento de las encuestas arrojó datos que permiten conocer algunos aspectos del vínculo de los usuarios con el estudio caso (FIGURA 7), entre los resultados más sobresalientes se encuentra el hecho que el $96 \%$ de los encuestados reside en el barrio de Almagro, mientras que solo el $4 \%$ vive en otro barrio. Entre quienes viven en el barrio de Almagro, el $67 \%$ lo hace a menos de tres cuadras de la Plaza, el $29 \%$ entre tres y cinco cuadras, y el $4 \%$ restante de los encuestados tiene su vivienda a más de cinco cuadras. El $96 \%$ de los encuestados utiliza la Plaza y, dentro de ellos, el $42 \%$ la usa de manera semanal, mientras que el $31 \%$ lo hace de forma diaria y el $27 \%$ solo durante los fines de semana. En relación con las actividades que los usuarios llevan a cabo en la Plaza, el 28\% manifestó que la utiliza como lugar de esparcimiento, el 18\% de los mismos se acerca a ella para comprar en la feria y el $14 \%$ para realizar deporte, mientras que el $13 \%$ lleva a sus niños a los juegos infantiles y el 13\% lleva a sus mascotas de paseo a la Plaza. Por último, el $6 \%$ de los

7. Resultados más relevantes arrojados por las encuestas. Fuente: elaboración propia, 2019.

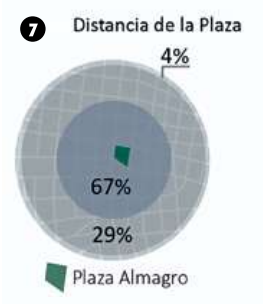

encuestados la usa como punto de encuentro, el $5 \%$ solo para el tránsito hacia otro sitio, y solo el $4 \%$ se dirige a la Plaza para asistir a eventos culturales. El $50 \%$ de los encuestados considera que el espacio para realizar la actividad es correcto, entre las causas mencionadas se encuentran el buen estado general del espacio, la vegetación, la presencia de un canil, de la feria y de los juegos. Dentro del 50\% restante, las causas principales por las cuales no consideran que el espacio sea el adecuado son: dimensiones insuficientes, escasez de césped, poco mantenimiento y presencia de feriantes no regulados. Frente a la pregunta sobre qué aspectos mejoraría de la Plaza, las respuestas más frecuentes fueron la vegetación, la limpieza y el mobiliario.

\section{DIAGNÓSTICO}

La Plaza Almagro constituye un espacio público importante dentro del barrio de Almagro y de la Comuna 5 debido, por un lado, a la escasez de estos $y$, por el otro, por la alta densidad poblacional y, en consecuencia, la baja densidad de $\mathrm{m}^{2}$ de espacio verde por habitante.

Con el objetivo de poder puntualizar los elementos de la Plaza que influyen en la problemática de superposición de usos, se detectaron los aspectos negativos y positivos de la Plaza Almagro en base a cuatro variables (Pinto y Remesar, 2015): usos y actividades, sociabilidad, forma y acceso. Estas variables tienen en cuenta aspectos del espacio que permiten determinar el nivel de cohesión del mismo, tanto de las distintas partes que lo conforman como con su entorno físico y los actores que en él participan, ya que implica la detección de aspectos positivos y negativos tanto en los elementos físicos, como la forma y el nivel de accesibilidad, así como en los funcionales y sociales.

Como aspectos negativos más relevantes en referencia a los usos y actividades, es decir, si los distintos sectores de la Plaza promueven o no un determinado uso del espacio, y si este uso es colectivo o individual, se advierte que, en base a las observaciones participantes llevadas a cabo, se encuentra compartimentada en distintas áreas con usos específicos y mixtos, con poca presencia de espacios multifuncionales cuando, según los resultados arrojados por las encuestas, el $28 \%$ de los encuestados la usa como lugar de esparcimiento. A su vez, hay una gran disparidad en cuanto a intensidad de usos dentro de los distintos sectores de la Plaza y escasos sectores que permitan el encuentro y la socialización, lo cual influye directamente en la problemática de sobreuso de espacios dentro de la Plaza.

De todos modos, se observó, como elemento positivo, que las áreas de usos específicos tienen la intención de fomentar en los vecinos del barrio la realización de actividades físicas, de entretenimiento y culturales, en su mayoría, de carácter grupal.

En contraposición, se detectó como elemento más significativo en cuanto a la sociabilidad dentro del espacio, relacionada con el tipo de usuario que asiste a la Plaza, que esta propicia su interacción y, así, la cohesión de 
la comunidad, el alto grado de apropiación por parte de los vecinos, que conforman la mayor parte de los usuarios de la Plaza según las encuestas realizadas, ya que el $96 \%$ de los encuestados vive en el barrio de Almagro y el $67 \%$ de ellos lo hace a menos de tres cuadras de la Plaza, mostrando estos usuarios una gran diversidad en cuanto a edades y propósito de concurrencia.

En cuanto a la forma, es decir, si el espacio puede ser fácilmente leído como una continuidad dentro del lugar donde se encuentra emplazado y cómo es la distribución interna del espacio y las dimensiones de cada subespacio: se reconoce como aspecto positivo que, al encontrarse emplazada en una manzana rodeada por edificios de viviendas y comercios, la Plaza se lee fácilmente como una unidad con función de espacio público. Como aspectos negativos, en cambio, se determinó que solo hay sectores puntuales, como la zona central, que estimula la realización de distintos tipos de actividades, luego el resto de las áreas tienen funciones predeterminadas específicas como los juegos para niños, cancha deportiva, canil y posta deportiva, y el $50 \%$ de los encuestados consideran que el espacio donde realiza sus actividades no es correcto para tal fin. Además, en base a las observaciones realizadas se detectó que el usuario es inducido a caminar por senderos específicos de manera radial y concéntrica, no permitiendo un recorrido libre y los espacios de descanso, dados por la presencia de mobiliario, mayormente existen solo sobre los senderos.

En relación a los accesos se pudo determinar, a través del análisis planimétrico y de las observaciones realizadas, que la Plaza se encuentra dentro una zona residencial y comercial muy importante dentro del barrio de Almagro, con varias líneas de colectivo que transitan por sus calles perimetrales y, por lo tanto, el acceso a la zona es sencillo. Pero, el fuerte borde dado por la reja perimetral no permite el libre acceso a la Plaza por cualquiera de sus lados, ya que existen cuatro entradas ubicadas su alrededor por donde se puede ingresar. Asimismo, dentro de la Plaza, el ingreso tanto a los sectores con funciones específicas como a las áreas de estar con césped, es restringido también por la presencia de rejas, de menor altura que la perimetral, pero que dificultan el acceso a cada subespacio.

\section{CONCLUSIONES}

Como aspectos destacados del análisis realizado sobre la Plaza Almagro se pudieron detectar y localizar las actividades que allí se desarrollan, mediante el análisis planimétrico, la observación participante y las encuestas realizadas, se pudieron determinar la gran variedad de actividades que los usuarios realizan en la Plaza, que no se ven reflejados en el diseño actual de la misma, como esparcimiento, deportes, paseo de mascotas, llevar niños a los juegos infantiles, entre otras. A su vez, a través de las observaciones, se logró analizar la superposición de esos usos en el espacio verde, pudiendo precisar así los momentos de mayor saturación que se desarrollan durante los fines de semana y en la época estival, y determinar las zonas con mayor conflicto en el mismo sentido, lo cual determinó, también, que las principales actividades superpuestas son las infantiles con las deportivas, las de descanso y el paseo de mascotas.

Este análisis de usos dentro de la Plaza fue de gran importancia para la realización del diagnóstico, que permitió evidenciar los aspectos que influyen en la problemática de sobreuso del espacio. En este sentido, se puede concluir que la Plaza es un espacio público con una alta apropiación por parte de los vecinos, y cuenta con una interesante diversidad de usuarios en cuanto al grupo etario al que pertenecen, sumado a que se encuentra ubicada en una zona que funciona como un nodo de circulación relevante dentro del barrio debido a la oferta de transporte público en las inmediaciones que permiten la conexión con el resto de la Ciudad de Buenos Aires. Pero estas fortalezas del espacio se ven disminuidas debido a los aspectos negativos que posee, principalmente relacionados a la excesiva fragmentación de su espacio en compartimentos que, como arrojaron los resultados de las encuestas, no responden a las necesidades de los usuarios.

A su vez, hay sucesivas barreras que dificultan el acceso a las diferentes áreas y la movilidad fluida por toda la Plaza, generando que los espacios que no tienen un uso definido no puedan tampoco funcionar correctamente como áreas multifunción, donde poder realizar distintas actividades dependiendo del día, horario y necesidad de los usuarios. Esto deriva en un desbalance en la intensidad de usos de cada uno de esos sectores de la Plaza, generando zonas frecuentemente en desuso y otras donde la intensidad de uso es tan alta que derivan en una saturación del espacio verde, generando complicaciones e incomodidad para los usuarios.

Por lo expuesto anteriormente, resulta imprescindible para los vecinos del barrio contar con un espacio verde público que contribuya al mejoramiento de su calidad de vida donde todos puedan encontrar un lugar de recreación. Es por esto que es necesario un mayor grado de cohesión de la Plaza, tanto a nivel físico como social y funcional, que permita un mejoramiento en la convivencia de los usuarios y sus distintas actividades, y una optimización del espacio físico que se traduzca en mayor confort, libertad de uso y circulación para los asistentes a la Plaza.

La problemática del colapso de actividades y usuarios en la Plaza Almagro, objeto del presente trabajo, es un conflicto contemporáneo que se extiende a múltiples espacios verdes públicos urbanos. Por este motivo, el presente trabajo buscó, a través del análisis de un caso de estudio, revelar la importancia de analizar en profundidad la configuración de las áreas verdes urbanas y reflexionar acerca de cuáles deberían ser los criterios y la relevancia de la participación ciudadana al momento de realizar modificaciones en las mismas que contribuyan a solucionar estas problemáticas de saturación del espacio verde público. 
La planificación y el diseño de los espacios verdes públicos urbanos deberían ser el resultado de políticas públicas inclusivas orientadas al mejoramiento de la calidad de vida de todos los ciudadanos.

Las distintas realidades sociales, políticas y económicas no son estáticas, sino que, por el contrario, se modifican a lo largo del tiempo, y los espacios públicos urbanos debieran reflejar estos cambios para que puedan responder a las necesidades de los usuarios en cada momento histórico mediante la construcción de espacios públicos de calidad, inclusivos y participativos que promuevan la cohesión social.

\section{REFERENCIAS BIBLIOGRÁFICAS}

Anduze Rivero, V. R. (2019). La participación y

la apropiación del espacio público como fundamentos del derecho a la ciudad: dos estudios de caso al sur de Mérida, Yucatán. Península, 14(1), 29-50. Recuperado de http:// www.scielo.org. $\mathrm{mx} / \mathrm{scielo} \cdot \mathrm{php}$ ? script $=$ sci_ arttext\&pid=\$1870-57662019000100029\&lng $=$ es\&tlng $=$ es.

Blanco-Bello, R., \& Victoria-Cogollo, K. (2013). Los espacios públicos en sectores populares de Cartagena: lugares de encuentro y desencuentro. Entramado, 9(2), 176-179. Recuperado de http://www.scielo.org.co/scielo. php? script=sci_ artext\&pid= S1900-38032013000200012\&lng $=$ en\&tlng $=$ es.

Borja, J. (2014). Revolución urbana y derechos ciudadanos. Buenos Aires: Café de las ciudades.
Castillo-Villar, F. R. (2018). City branding and the theory of social representation. Bitácora Urbano Territorial, 28(1), 33-38. https://dx.doi. org/10.15446/bitacora.v28n1.52939.

Dollfus, O. (1990) El espacio geográfico. Barcelona: Oikos-Tau.

Duverger, M. (1996) Métodos de las Ciencias Sociales. Barcelona: Editorial Ariel.

Fernández, R. (2013). El espacio público en disputa: Manifestaciones políticas, ciudad y ciudadanía en el Chile actual. Psicoperspectivas, 12(2), 28-37. https://dx.doi.org/10.5027/psicoperspectivasVol12-Issue2-fulltext-278.

Gobierno de la Ciudad de Buenos Aires (2018a) Buenos Aires Ciudad. Buenos Aires. Superficie de espacios verdes por habitante por comuna. Ciudad de Buenos Aires. Años 2006/2018. Recuperado de: https://www.estadisticaciudad. gob.ar/eyc/? $p=27349$

Gobierno de la Ciudad de Buenos Aires (2018b) Buenos Aires Ciudad. Buenos Aires. Población total estimada por sexo, superficie y densidad poblacional según comuna. Ciudad de Buenos Aires. Años 2006/2018. Recuperado de https:// www.estadisticaciudad.gob.ar/eyc/? $p=76599$

Gobierno de la Ciudad de Buenos Aires (2019) Buenos Aires Ciudad. Buenos Aires. Obras Comunales. Recuperado de: https://www. buenosaires.gob.ar/comuna-5/obras-comunales

Gómez Carmona, G., \& Villar Calvo, A.J. (2014). Apropiación simbólica y reconfiguración identitaria del espacio urbano en Metepec, Estado de México. Nova scientia, 6(1 1), 268290. Recuperado de http://www.scielo.org. $\mathrm{mx} /$ scielo.php? script=sci_arttext\&pid=\$2007$07052014000100015 \& \operatorname{lng}=$ es\&tlng $=$ es .
Hernández Sampieri, R.; Fernández-Collado, C; Baptista Lucio, P. (2006). Metodología de la investigación. Editorial: McGraw Hill.

Jodelet, D. (1986) "La representación social: fenómenos, concepto y teoría". En: Moscovici, S. Psicología social, II. Barcelona: Paidós.

Lindón, A. (2006) "Capítulo 16, Geografías de la vida cotidiana". En: Hiernaux, D. y Lindón, A. y (dirs.). Tratado de geografía humana. Universidad Autónoma Metropolitana - Iztapalapa: Anthropos Editorial, pp. 356-400.

Narváez-Muelas, D. A. (2019). Privatización del espacio público verde en la era neoliberal. El Club Tequendama. En Cali, Colombia. Bitácora Urbano Territorial, 29(1), 121-130. https:// dx.doi.org/10.15446/bitacora.v29n1 .69048.

Páramo, P., \& Burbano Arroyo, A. M. (2011). Género y espacialidad: análisis de factores que condicionan la equidad en el espacio público urbano. Universitas Psychologica, 10(1), 61-70. Recuperado de http://www.scielo.org. $\mathrm{co} /$ scielo.php? script=sci_arttext\&pid $=$ S165792672011000100006\&lng =en\&tlng =es.

Pinto, A.J., Remesar, A. (2015). Urban cohesion: a public space network assessment. On the waterfront, 39 (2) ISBN: 1139-7365.

Roldán, D. (2018). Imaginarios y prácticas del espacio público. La Feria de Colectividades de Rosario, Argentina. Andamios, 15(38), 1 13-139. https:// dx.doi.org/10.29092/vacm.v15i38.654.

Santos, M. (1996) Metamorfosis del espacio habitado. Barcelona: Oikos-Tau. 